\title{
Effects of masker envelope irregularities on tone detection in narrowband and broadband noise maskers
}

\author{
Emily Buss, Joseph W. Hall III, and John H. Grose \\ Department of Otolaryngology/Head and Neck Surgery University of North Carolina School of \\ Medicine Chapel Hill, NC 27599
}

\begin{abstract}
Introducing coherent masker envelope modulation to frequency regions neighboring the signal frequency can reduce detection thresholds for a pure-tone signal. Verhey and Ernst (2009) reported that irregular masker modulation conferred greater benefit than regular modulation when the masker was broadband, but that there was no difference when the masker was narrowband. The present study evaluated two possible explanations for this result: one based on modulation adaptation and the other based on the introduction of relatively long-duration modulation minima in the irregular masker modulation condition. The first experiment replicated the results of Verhey and Ernst (2009), but also included conditions in which a 12.5-ms signal was presented in a 12.5ms modulation minimum, which was exempt from envelope jitter. The second experiment used a continuous masker and suspended jitter during epochs associated with either a $12.5-$ or $87.5-\mathrm{ms}$ signal. No benefit of masker envelope irregularity before or after the signal was observed in either experiment. These findings are inconsistent with an explanation based on modulation adaptation, implicating instead the introduction of relatively long-duration modulation minima in the large masking release obtained for a long-duration signal in an irregularly modulated masker.
\end{abstract}

\section{Keywords}

masking; masking release; envelope modulation; adaptation; psychoacoustics

\section{Introduction}

Detection of a pure-tone signal in a fluctuating masker can be improved by increasing the
bandwidth of that masker, provided that the masker envelope is coherently amplitude
modulated across frequency. This phenomenon is referred to as comodulation masking
release (CMR). In a recent study, Verhey and Ernst (2009) compared CMR for periodic
amplitude modulation (AM) and for irregular AM. The envelope of the periodic stimulus
was a 40-Hz square wave, smoothed with a 5-ms Hanning window, and the envelope of the
irregular stimulus was constructed by temporally jittering the level transitions of the
smoothed 40-Hz square wave. One motivation for the Verhey and Ernst study was to test a
computational model of CMR based on the output of a modulation filterbank (Verhey et al.,
1999), which predicts greater masking release for periodic than for irregular masker
envelopes. Contrary to this prediction, Verhey and Ernst (2009) reported that thresholds in a

(C) 2012 Elsevier B.V. All rights reserved.

Corresponding author: Emily Buss, ebuss@med.unc.edu, 0119199668926.

Publisher's Disclaimer: This is a PDF file of an unedited manuscript that has been accepted for publication. As a service to our customers we are providing this early version of the manuscript. The manuscript will undergo copyediting, typesetting, and review of the resulting proof before it is published in its final citable form. Please note that during the production process errors may be discovered which could affect the content, and all legal disclaimers that apply to the journal pertain. 
broad (3750-Hz-wide) masker fell as the temporal jitter applied to the masker envelope increased from $0 \%$ to $20 \%$ of the modulation period. In contrast, thresholds were constant across masker jitter conditions when the masker was a narrow (128-Hz-wide) band of noise. Two possible explanations were offered for the finding of better performance when the broadband masker AM was jittered: 1) better across-channel cues associated with the relatively long-duration AM minima introduced by the modulation jitter, and 2) reduced modulation adaptation with the jittered masker envelope. These two possibilities are considered in turn.

In the study of Verhey and Ernst (2009), the introduction of temporal jitter resulted in masker modulation minima that were of non-uniform duration. In the regular modulation condition, the masker envelope was a smoothed $40-\mathrm{Hz}$ square wave, with a $50 \%$ duty cycle; as such, modulation minima were $12.5 \mathrm{~ms}$ in duration. In the maximal (20\%) jitter condition, masker envelope transitions were jittered by $\pm 20 \%$ of the modulation period; the duration of modulation minima ranged from 2.5 to $22.5 \mathrm{~ms}$, with a median value of $12.5 \mathrm{~ms}$ (corresponding to $40 \mathrm{~Hz}$ ). The pure-tone signal used by Verhey and Ernst (2009) was 200 $\mathrm{ms}$ in duration, measured from the half-rise points of the $50-\mathrm{ms}$ onset and offset ramps ${ }^{1}$, so it spanned approximately 8 modulation periods of the masker envelope. In the jittered-AM masker, it is likely that one or more of the masker minima occurring during the signal presentation would have been substantially longer than the 12.5 -ms minima in the periodic modulation condition. Previous work has shown the importance of modulation minima for tone detection in a coherently modulated masker that spans multiple auditory filters, with some indication that signal detection is dominated by 'listening in the valleys' of the masker envelope (Buus, 1985; Buus et al., 1996; Grose and Hall, 1989). The ability to benefit from masker modulation falls off with increasing modulation rate (Carlyon et al., 1989), an effect that has been attributed to the limits of temporal resolution in this task (Bacon et al., 1997). It is therefore possible that the occasional long-duration minima in the jittered-AM masker could have provided particularly informative cues in the stimuli used by Verhey and Ernst (2009). The dominant limit to temporal resolution is forward masking (Moore et al., 1988), and it is possible that a reduction in the effects of forward masking was responsible for the better performance in jittered-AM conditions.

Verhey and Ernst (2009) posed two arguments against the possibility that forward masking played a role in the effect of masker envelope jitter. First, the model tested in that report incorporates the effects of forward masking, and yet it does not predict better performance in the jittered than the periodic comodulated masker conditions. Second, no effects of masker envelope jitter were observed for narrowband noise maskers, where temporal resolution was also presumed to be an important factor. Verhey and Ernst (2009) argued that withinchannel temporal resolution could not account for the effects of jitter, but that there could be an additional limit to temporal resolution that is specific to modulation-based, acrosschannel processes.

Another explanation proposed by Verhey and Ernst (2009) to account for the beneficial effects of modulation jitter in the broadband masker conditions is related to modulation adaptation. Modulation adaptation can be demonstrated by measuring AM detection thresholds before and after exposure to an adaptor stimulus with pronounced AM. Using this method, Kay and Matthews (1972) found that adaptation increased AM detection thresholds by a factor of three. Modulation adaptation is maximized when the adaptor and the test stimulus share the same carrier frequency and modulation rate (Richards et al., 1997;

\footnotetext{
${ }^{1}$ Verhey and Ernst (2009) reported the total signal duration as 250-ms, including 50-ms onset and offset ramps. Half-rise durations are reported in the present study, in part to facilitate estimates of temporal integration for conditions that differed in onset/offset ramp duration.
} 
Wojtczak and Viemeister, 2003). Whereas most studies of AM adaptation have measured AM detection thresholds, Wojtczak and Viemeister (2003) observed similar effects for supra-threshold AM using a modulation-matching procedure. In that task, listeners adjusted the modulation depth of a 4-kHz comparison tone to match the subjective modulation depth of a 1-kHz standard. Under some conditions, prior exposure to a 1-kHz AM adaptor decreased the perceived AM depth of the standard, as reflected in the reduced AM depth of the comparison tone at the point of subjective equality. Adaptation was largest when the AM rate of the adaptor matched the AM rate of the standard. These results indicated a carrierspecific reduction in the subjective modulation depth after adaptation. One interpretation of modulation adaptation is that stimulation of neural channels tuned to AM leads to fatigue (Kay and Matthews, 1972; Tansley and Suffield, 1983). This interpretation is brought into question, however, by results indicating that adaptation to AM depends on attention (Kawashima, 2009) and can be eliminated with extended practice (Bruckert et al., 2006).

The idea that AM is coded in channels that are tuned to modulation rate has been formalized in models incorporating a modulation filterbank (Dau et al., 1997), including models of CMR (Verhey et al., 1999). Modulation adaptation could reduce sensitivity to a pure tone in a comodulated masker with a regular envelope in at least two ways. First, degradation in the internal representation of AM in the auditory channel centered on the signal frequency could reduce a listener's ability to detect a reduction in AM depth associated with the addition of a signal. Second, adaptation could reduce the efficacy of envelope-based across-channel processes.

The goal of the present study was to examine further the effects of masker envelope irregularity, with an emphasis on discriminating between an explanation based on greater ability to benefit from relatively long-duration AM minima and one based on adaptation. The primary strategy used to control effects related to temporal resolution was to suspend masker envelope jitter during presentation of the signal, such that energetic masking was comparable across periodic and irregular masker AM conditions. For example, in the first experiment the signal was $12.5 \mathrm{~ms}$ in duration, matching the duration of a single envelope minimum of a $40-\mathrm{Hz}$ square-wave masker envelope. In the associated irregular envelope condition, the envelope minimum coinciding with the signal was excluded from jitter and was always $12.5 \mathrm{~ms}$ in duration. Whereas Verhey and Ernst (2009) used a 200-ms signal, the motivation for using relatively brief signals in the present experiments was to avoid suspending masker AM jitter for long periods of time. The premise was that masker envelope jitter would affect thresholds for brief and long signals to a comparable degree. Duration-specific effects would undermine this approach, however, so special attention to duration is warranted in evaluating the results.

Increasing the duration of a relatively brief signal improves thresholds by approximately 3 $\mathrm{dB}$ per doubling of duration at $1000 \mathrm{~Hz}$ under many listening conditions (Gerken et al., 1990), a result described as temporal integration. Similar patterns of temporal integration have been observed whether the signal is presented in one burst or multiple sequential bursts (Gerken et al., 1990), even if the intervals between signal bursts are filled with masking noise (Viemeister and Wakefield, 1991) ${ }^{2}$. These observations support the idea that temporal integration may be based on the selective combination of 'multiple looks' at the signal, rather than the accumulation of power over a relatively long time window (e.g., leaky integrator model). Temporal integration is considerably less than $3 \mathrm{~dB}$ per doubling if the

\footnotetext{
2 In a multiple-looks model, the provision of two independent 'looks' at the signal is expected to improve d' by a factor of $\sqrt{ } 2$. For the conditions in Viemeister and Wakefield (1991), this corresponded to a threshold improvement of about $1.5 \mathrm{~dB}$. Temporal integration for a pair of brief signals separated by a 100-ms masker was approximately $2.5 \mathrm{~dB}$. This result was described as consistent with an optimal combination of multiple looks ( $1.5 \mathrm{~dB}$ per doubling), but it is also quantitatively consistent with previous literature showing 3 $\mathrm{dB}$ per doubling.
} 
signal is spectrally broad relative to a critical band (van den Brink and Houtgast, 1990), and temporal integration varies across individual listeners and particular stimulus conditions (Gerken et al., 1990).

Several studies have noted particularly large temporal integration when the masker is a set of comodulated narrow bands of noise separated in frequency (Buus, 1999; Schooneveldt and Moore, 1989), although this is not always found (McFadden, 1986). Temporal integration that is greater than $3 \mathrm{~dB}$ per doubling could be interpreted as indicating that detection in a comodulated masker differs for brief and long signals. Alternatively, it could reflect the variation in the duration or depth of masker modulation minima over time for narrowband noise maskers, such that longer signals are more likely than short signals to coincide with 'the best' minimum in the sample. This latter factor would not be expected to affect results in the periodic-AM conditions of the present study; differences in modulation minima of a square-wave AM noise band over time would be small apart from effects of envelope jitter. The expectation was therefore that comparable temporal integration would be observed for the steady (unmodulated) and periodic-AM masker conditions. Deviation in the expected temporal integration in AM maskers would cast doubt on the assumption that thresholds for short and long signals reflect the same underlying processes.

Both experiments reported below closely followed the methods of Verhey and Ernst (2009). The first experiment assessed the contributions of long-duration minima to the benefit associated with irregular masker AM by comparing results for a 200-ms signal, like that used by Verhey and Ernst, and a 12.5-ms signal, presented during a fixed, 12.5-ms modulation minimum. The second experiment followed this general paradigm but used a different form of envelope jitter and continuous masker presentation, with the aim of maximizing possible effects related to adaptation. Results were obtained for a $12.5-\mathrm{ms}$ and a 87.5-ms signal, each presented during epochs of periodic modulation, to evaluate further the importance of signal duration in the effects under study. In both experiments, the benefit associated with periodic or regular modulation was determined with respect to threshold in an unmodulated noise, for both narrow and broad masker bandwidths; in the present study, the difference in threshold for steady and AM maskers (irrespective of masker bandwidth) is referred to as the modulated/unmodulated difference (MUD). The term CMR is reserved for the comparison between thresholds in the narrowband and broadband AM maskers, in which the introduction of coherent masker AM in auditory channels flanking the signal frequency is thought to improve performance ${ }^{3}$.

\section{Experiment 1}

The goals of the first experiment were to: 1) replicate the finding of lower thresholds with jittered than periodic AM applied to a broadband masker for a long-duration signal, as observed by Verhey and Ernst (2009), and 2) determine whether this effect could be obtained under conditions controlling for possible effects of temporal resolution.

\subsection{Listeners}

Thirteen listeners were recruited. One was excluded due to excessive threshold variability, with thresholds spanning a range of $29 \mathrm{~dB}$ in one condition. Two listeners were unable to finish the experiment, although the data they provided were consistent with those for the other listeners. The remaining group of ten listeners ranged in age from 29 to 59 years of age (mean $37 \mathrm{yrs}$ ). All had pure tone thresholds of $20 \mathrm{~dB}$ HL or better at octave frequencies $250-8000 \mathrm{~Hz}$ (ANSI, 2010), and none of the listeners reported a history of ear disease.

\footnotetext{
${ }^{3}$ This convention is different from that used by Verhey and Ernst (2009), where the benefit of masker AM was identified as a CMR for both narrowband and broadband maskers.
} 
Seven listeners were highly experienced in psychoacoustics, having participated in at least one previous study of CMR. One author provided data in this experiment.

\subsection{Procedures}

Stimuli and procedures closely followed those of Verhey and Ernst (2009). The masker was a band of noise that was either broadband ( 250 to $4000 \mathrm{~Hz}$ ) or narrowband (923 to 1051 $\mathrm{Hz}$ ). These bands were generated in the frequency domain by setting the magnitude of components outside the desired passband to zero. The masker was either steady or amplitude modulated via multiplication with a smoothed square-wave function ${ }^{4}$. In the periodic-AM condition, the envelope was a $40-\mathrm{Hz}$ square wave with $50 \%$ duty cycle, which was smoothed via convolution with a 5-ms Hanning window; this envelope alternated between 12.5-ms maxima and minima. In the jittered-AM condition, the periodic envelope was modified by delaying or advancing each level change (onset or offset) over the range of $\pm 20 \%$ of the modulation period, based on random draws from a uniform distribution. As a result, envelope minima and maxima could take on values between 2.5 and $22.5 \mathrm{~ms}$. Because the underlying rate was fixed $(40 \mathrm{~Hz})$, the longest- duration minima were preceded and followed by relatively short-duration maxima and vice versa. All maskers had a duration of $300 \mathrm{~ms}$ at the half-rise points, and they were gated on and off with 50-ms raised-cosine ramps. Narrowband and broadband masker levels were $50 \mathrm{~dB}$ SPL and $65 \mathrm{~dB}$ SPL, respectively.

The signal was a $986-\mathrm{Hz}$ pure tone, temporally centered in the masker. In the 200- ms signal condition, the signal was gated on and off with 50-ms raised-cosine ramps, and its duration was $200 \mathrm{~ms}$ at the half-rise points. This is equivalent to the signal used by Verhey and Ernst (2009). In the 12.5-ms signal condition, the signal had the same duration as a single periodic-AM minimum $(12.5 \mathrm{~ms})$, with onset and offset smoothed via convolution with a 5ms Hanning window. This produced signal gating that was complementary to the gating defining masker envelope transitions. In the long-dip signal condition, the signal duration was equal to the duration of the longest masker modulation minimum in the corresponding jittered-AM masker sample, with which it coincided. When the long-dip signal was presented in the steady masker, a jittered-AM masker sample was generated with the sole purpose of determining an appropriate signal duration. Thresholds for the long-dip signal were not estimated in the periodic-AM masker.

Unlike the study of Verhey and Ernst (2009), the jittered-AM envelope always contained at least one minimum that was exactly $12.5 \mathrm{~ms}$ in duration, matching the modulation minima in the periodic-AM envelope. Examples of these stimuli are shown in Figure 1, with signals at the top of the panel and maskers at the bottom of the panel. In the 200-ms signal condition, this minimum occurred at a random time point in the masker array ${ }^{5}$. In the 12.5ms signal condition, the masker array was rotated so that the 12.5 -ms minimum occurred in the temporal center of the masker, coincident with the signal when it was present. In the long-dip signal condition, the masker was rotated so that the longest-duration minimum in the sample occurred in the temporal center of the masker, coincident with the signal, and the $12.5-\mathrm{ms}$ minimum occurred at a random time point. A set of 1000 jittered-AM maskers was generated to determine the duration of the 'longest-duration' minima. In this set, the longestduration minimum had a median value of $19.0 \mathrm{~ms}$, with half of the samples falling between 17.7 and $20.0 \mathrm{~ms}$.

\footnotetext{
${ }^{4}$ The narrowband conditions of Experiment 1 corresponded to those labeled "NB2" by Verhey and Ernst (2009). Filtering was applied before, not after, modulation.

${ }^{5}$ In the 200-ms signal condition, the 12.5-ms masker envelope minimum could occur before or after the signal interval.
} 
All stimuli were generated in MATLAB, with novel samples of noise and jittered envelopes on each presentation. Stimulus arrays were played out of a real-time digital signal processor (RP2; Tucker-Davis Technologies), routed through a headphone buffer (HB7, Tucker-Davis Technologies), and presented diotically over circumaural headphones (Sennheiser, HD 265). The sampling rate was $12207 \mathrm{~Hz}$.

A three-alternative forced-choice task was used, with 350-ms listening intervals and 500-ms inter-stimulus intervals. The signal was equally likely to occur in each of the three listening intervals. Listeners indicated the signal interval using a handheld response box, and visual feedback followed each response. The masker level was fixed, and the signal level was adjusted based on a two-down, one-up tracking rule that estimated the signal level associated with $71 \%$ correct (Levitt, 1971). These adjustments were initially made in steps of $8 \mathrm{~dB}$; steps were reduced to $4 \mathrm{~dB}$ after the second track reversal and to $2 \mathrm{~dB}$ after the fourth track reversal. A track continued until a total of 10 reversals had been obtained. Threshold estimates were computed as the mean signal level at the last 6 track reversals. At least three such estimates were obtained for each condition, with a fourth in cases where the first three spanned a range of $3 \mathrm{~dB}$ or more. Listeners completed all threshold estimates in a condition before moving on to the next condition. Conditions were visited in quasi-random order, with the following exception; eight listeners completed the broadband conditions before the narrowband conditions, and two completed the narrowband conditions before the broadband conditions. In four cases, thresholds in a single condition varied by $8 \mathrm{~dB}$ or more for an individual listener, and in those cases the associated data were replaced at the end of the experiment. For two listeners, thresholds for the 12.5 -ms signal were $\sim 10 \mathrm{~dB}$ higher than those for the remaining listeners; completing these conditions a second time brought the performance of these listeners more in line with that of other listeners, and the second set of values is reported here. Replacing these data did not change the general outcome of the experiment. All simple effects tests used a Bonferroni correction.

\subsection{Results}

The pattern of results was consistent across individual listeners, so the mean data are shown in Figure 2. Results for each signal condition are shown in a different panel. Thresholds obtained with the narrowband masker are shown with filled triangles and dotted lines, and thresholds obtained with the broadband masker are shown with open circles and solid lines. Thresholds for signals in the steady masker, shown with horizontal lines, are described first.

2.3.1 Thresholds for Signals in the Steady Masker-Thresholds for signals in the steady maskers tended to improve with signal duration. Recall that temporal integration for detection of a tone in a steady noise is approximately $3 \mathrm{~dB}$ per doubling of duration under many conditions (Garner and Miller, 1947). A 12-dB improvement is therefore expected when the signal duration is increased from $12.5-\mathrm{ms}$ to $200-\mathrm{ms}$. This is consistent with the temporal integration observed for the broadband masker $\left(12.8 \mathrm{~dB} ; \mathrm{t}_{9}=1.85, \mathrm{p}=0.098\right)$, while integration for the narrowband masker was significantly less than expected $\left(8.0 \mathrm{~dB} ; \mathrm{t}_{9}=5.13\right.$, $\mathrm{p}=0.001$ ). One reason for the relatively modest temporal integration observed for the narrowband masker could be related to spectral splatter. The spectrum of the 12.5 -ms signal was only approximately $13 \mathrm{~dB}$ down at the limits of the narrow (128-Hz-wide) masker. Cues related to splatter would have had less of an effect on thresholds in the 200-ms signal conditions, due to the longer onset/offset ramps ( $50 \mathrm{~ms}$ vs. $5 \mathrm{~ms}$ ).

2.3.2 Effects of Masker AM (MUD)—The introduction of masker AM consistently reduced thresholds for signals in the broadband masker. In contrast, thresholds for signals in the narrowband masker improved only for the 200-ms signal conditions; masker AM had no beneficial effect for the 12.5 -ms or long-dip signal conditions. One reason for the absence of 
a MUD for brief signals in the narrowband masker could be related to splatter. Whereas splatter associated with signal ramps could provide a detection cue in a steady masker, the splatter associated with masker AM could mask that cue in both the periodic-AM and jittered-AM masker conditions.

Values of the MUD in the 200-ms signal conditions are considered first. For the broadband masker, the MUD was larger for the jittered-AM $(15.2 \mathrm{~dB})$ than for the periodic-AM (12.1 $\mathrm{dB})$ masker condition. For the narrowband masker, there was a somewhat smaller difference in the MUD obtained in the jittered-AM $(13.1 \mathrm{~dB})$ and the periodic-AM $(11.6 \mathrm{~dB})$ masker conditions. A repeated-measures analysis of variance (ANOVA) was performed to assess this pattern of results, with factors masker bandwidth (broad, narrow) and masker envelope regularity (jittered-AM, periodic-AM). There was no effect of bandwidth $\left(\mathrm{F}_{1,9}=1.57\right.$, $\mathrm{p}=0.242$ ). There was, however, a significant effect of masker envelope regularity $\left(\mathrm{F}_{1,9}=66.05, \mathrm{p}<0.001\right)$, and an interaction between bandwidth and envelope regularity $\left(\mathrm{F}_{1,9}=9.80, \mathrm{p}=0.012\right)$. Simple effects tests indicated a significant effect of masker AM jitter for both the broadband masker $(3.1 \mathrm{~dB} ; \mathrm{p}<0.001)$ and the narrowband masker $(1.5 \mathrm{~dB}$; $\mathrm{p}=0.009$ ). These results broadly replicate those of Verhey and Ernst (2009), with the exception of the modest but significant effect of masker envelope regularity for the narrowband masker.

The pattern of results for the 12.5 -ms signal was very different from that for the 200-ms signal. For the broadband masker, the MUD was comparable for the jittered-AM (14.7 dB) and the periodic-AM $(13.6 \mathrm{~dB})$ masker conditions. For the narrowband masker, the mean values of the MUD were negative for both the jittered-AM $(-2.7 \mathrm{~dB})$ and the periodic-AM $(-1.2 \mathrm{~dB})$ masker conditions. The negative MUD for a brief signal in a narrowband masker could be due to the availability of a splatter cue in the steady but not the AM masker conditions. Another possibility is that the introduction of a modulation minimum coincident with a brief signal degrades cues related to peak stimulus intensity. A repeated-measures ANOVA indicated a main effect of bandwidth $\left(\mathrm{F}_{1,9}=214.40, \mathrm{p}<0.001\right)$, no effect of envelope regularity $\left(\mathrm{F}_{1,9}=0.08, \mathrm{p}=0.788\right)$, and a significant interaction $\left(\mathrm{F}_{1,9}=7.41, \mathrm{p}=0.024\right)$. Simple effects tests were performed to assess this interaction. The effect of masker AM jitter was not significant for the broadband masker $(1.1 \mathrm{~dB} ; \mathrm{p}=0.361)$, but there was a non-significant trend for higher thresholds in the jittered-AM than the periodic-AM narrowband maskers $(-1.6 \mathrm{~dB} ; \mathrm{p}=0.086)$.

Results of the 200-ms signal conditions replicated the greater benefit of masker AM in the broadband masker for irregular envelopes observed by Verhey and Ernst (2009). This did not occur for the 12.5-ms signal, which was coincident with a 12.5-ms AM minimum; in this condition, masker envelope jitter before and after signal presentation did not significantly improve performance, as would be expected if adaptation were responsible for the beneficial effects of masker envelope irregularity in the 200-ms signal conditions. The long-dip signal condition was designed to evaluate the role of longer-than-average modulation minima in the jittered-AM, 200-ms signal condition. For the narrowband masker, the MUD for the long-dip signal was not significantly different from zero $\left(0.67 \mathrm{~dB} ; \mathrm{t}_{9}=0.73, \mathrm{p}=0.483\right)$, similar to results of the $12.5-\mathrm{ms}$ signal conditions. For the jittered-AM broadband masker, however, the MUD for the long-dip signal condition was $22.9 \mathrm{~dB}$, which is about $8 \mathrm{~dB}$ greater than the MUD in comparable 12.5-ms and 200-ms signal conditions. These results are consistent with the idea that the effect of masker envelope jitter for the 200-ms signal was due to the introduction of relatively long AM minima, which reduced thresholds for signals in the broadband but not the narrowband masker.

2.3.3 Effects of AM Masker Bandwidth (CMR)—In the present study, CMR was defined as the difference between thresholds obtained with narrowband and broadband 
maskers. For the 200-ms signal, the CMR was modest, with means of $3.3 \mathrm{~dB}$ for the jittered$\mathrm{AM}$ and $1.7 \mathrm{~dB}$ for the periodic-AM maskers. The CMR was substantially larger in the 12.5ms signal conditions, with means of $13.9 \mathrm{~dB}$ for the jittered-AM and $11.2 \mathrm{~dB}$ for the periodic-AM maskers. A repeated-measures ANOVA was performed, and there was a main effect of both duration $\left(\mathrm{F}_{1,9}=52.58, \mathrm{p}<0.001\right)$ and envelope regularity $\left(\mathrm{F}_{1,9}=12.21, \mathrm{p}=0.007\right)$, but no interaction $\left(\mathrm{F}_{1,9}=1.27, \mathrm{p}=0.289\right)$. The $\mathrm{CMR}$ was greater in the jittered-AM than the periodic-AM masker conditions for both bandwidths, but for different reasons. For the 200ms signal conditions, in which modulation jitter continued during signal presentation, the larger CMR in the jittered-AM condition was due to relatively good performance with the broadband masker. For the 12.5 -ms signal conditions, in which modulation jitter was suspended during signal presentation, the larger CMR in the jittered-AM condition was probably affected by relatively poor performance in the narrowband jittered-AM condition. This latter point is weakly supported by the findings of no effect of envelope regularity for the broadband masker and a statistical trend for better performance in the periodic-AM narrowband masker with the 12.5 -ms signal.

2.3.4 Effects of Signal Duration in AM Maskers-Temporal integration for a signal in a broadband masker was $11.3 \mathrm{~dB}$ for the periodic-AM and $13.3 \mathrm{~dB}$ for the jittered-AM condition. These values represent approximately $3 \mathrm{~dB}$ per doubling of signal duration (12 $\mathrm{dB})$. If integration were acting on cues associated with listening in the dips, then a 12-dB decrease in threshold would represent $4 \mathrm{~dB}$ per doubling in duration (1 vs 8 minima).

The signal in the long-dip condition had a median duration of $19 \mathrm{~ms}$, and yet thresholds in this condition were $10.7 \mathrm{~dB}$ below those for the jittered-AM, 12.5-ms signal. This finding is broadly consistent with effects of forward masking, in that the longer AM masker minimum containing the long-dip signal is associated with greater decay of forward masking than the minimum coincident with the 12.5 -ms signal. It is difficult to make a direct comparison between results in the long-dip and 200-ms signal conditions for the jittered-AM masker, due to the random distribution of modulation minima. Nevertheless, the fact that long-dip and 200-ms thresholds in the jittered-AM masker differed by only $2.6 \mathrm{~dB}$ is consistent with the idea that detection of the 200-ms signal was dominated by cues associated with the longer-duration modulation minima.

\subsection{Discussion}

The results for the 200-ms signal in the broadband masker replicated those of Verhey and Ernst (2009), showing greater benefit in the jittered-AM than in the periodic-AM conditions; this effect was approximately $3 \mathrm{~dB}$ in both datasets. Whereas a similar pattern of results was obtained with the narrowband masker in the present study, the benefit associated with jittered-AM was significantly larger for the broadband than for the narrowband masker. In the jittered-AM condition, some masker AM minima were longer than the median value of $12.5 \mathrm{~ms}$, and some were shorter. The brief signal conditions were designed to test the contribution of the relatively long minima by suspending envelope jitter for one period of masker modulation. Masker AM jitter did not reduce thresholds in the 12.5-ms signal conditions for either masker bandwidth, and there was a non-significant trend for jitter to impair performance for the narrowband masker. When the signal had the same duration as the single longest-duration modulation minimum in a jittered envelope, thresholds approached those for the 200-ms signal in the broadband masker, but not in the narrowband masker. These results are consistent with the idea that the presence of relatively long duration minima increased the MUD in the broadband jittered-AM, 200-ms signal condition. Relatively long modulation minima could be more beneficial than short minima due to limited temporal resolution, including within-channel factors (e.g., forward masking) and across-channel factors (e.g., 'listening in the valleys'). 
The present results for the 12.5-ms signal in periodic and jittered maskers are useful in assessing the possible influence of adaptation to modulation. An important finding was that when a 12.5 -ms signal was presented coincident with a 12.5 -ms modulation minimum, there was no effect of surrounding masker modulation regularity. That is, jittering the envelope before and after the time of the signal presentation did not affect performance. This result undermines the idea that adaptation to modulation played an important role in differences obtained for periodic and jittered maskers with the 200-ms signal. However, there are several factors in the design of this experiment that could have reduced the opportunity to observe effects of adaptation. The first is the use of gated stimuli. Tansley and Suffield (1983) observed that adaptation to modulation is a gradual process, taking 20-30 min to reach asymptote, and is quick to decay, with full recovery after $60 \mathrm{sec}$. Previous studies of adaptation have used adaptor durations on the order of 10 min (Richards et al., 1997; Wojtczak and Viemeister, 2003) or $12 \mathrm{sec}$ (Kay and Matthews, 1972). Short-term reductions in sensitivity to AM have been observed with stimuli less than $1 \mathrm{sec}$ in duration in studies of forward masking of modulation (Moore et al., 2009; Wojtczak and Viemeister, 2005), although it is unclear how this phenomenon relates to adaptation. Exposure to stimulus AM in the present paradigm was relatively limited, consisting of 300-ms segments, so it is possible that continuous masker presentation would increase the probability of observing effects of adaptation.

The second stimulus feature that could reduce the opportunity to see effects of adaptation is related to the method used to generate irregular envelopes. The envelope in the jittered-AM condition of Experiment 1 was generated by delaying or advancing envelope transitions in a $40-\mathrm{Hz}$ square-wave. This results in a prominent $40-\mathrm{Hz}$ modulation even in the jittered-AM condition, as illustrated in the lower left panel of Figure 3. This form of jitter might therefore offer a more modest reduction in adaptation than other forms of envelope jitter that are dominated to a lesser extent by a single modulation frequency.

\section{Experiment 2}

The primary purpose of Experiment 2 was to assess the effect of masker envelope jitter under conditions more likely to show effects of adaptation. To that end, the masker was continuous, and envelope jitter was designed to more broadly distribute envelope spectral components, as illustrated in the lower right panel of Figure 3. In this experiment, envelope jitter was introduced by randomly selecting the modulation period on a cycle-by-cycle basis. Masker AM was either periodic at $40 \mathrm{~Hz}$, or it was jittered, with the duration of each AM maximum and subsequent minimum of each period randomly selected from the range 2.5 to $22.5 \mathrm{~ms}$. This range was the same as that used in Experiment 1, although jittering the duration for each period eliminated the peak in the envelope spectrum at $40 \mathrm{~Hz}$ that was present in the stimuli of the previous experiment. Another advantage of this method is that each modulation minimum was equal in duration to the preceding maximum. As a result, suspending envelope jitter during the signal presentation in the irregular envelope conditions equated both simultaneous and forward masking with that for the regular envelope conditions.

A secondary goal of this experiment was to examine further the possible effect of signal duration on the ability to benefit from periodic and jittered masker AM. The premise of Experiment 1 was that the effect of masker envelope jitter would be the same for brief and long signals in all respects other than the availability of longer-than-average modulation minima in the 200-ms signal conditions. Experiment 2 assessed possible effects related to signal duration more directly by including two signal durations (12.5 and $87.5 \mathrm{~ms})$, with modulation jitter suspended during signal presentation in both cases. 


\subsection{Listeners}

Listeners were nine adults, ranging from 29 to 59 years of age (mean 38 yrs). All had pure tone thresholds of $20 \mathrm{~dB}$ HL or better at octave frequencies $250-8000 \mathrm{~Hz}$ (ANSI, 2010), and none reported a history of ear disease. All of these listeners were highly experienced in psychoacoustics; all but one had previously completed Experiment 1.

\subsection{Procedures}

Stimuli were identical to those of Experiment 1 unless otherwise specified. The signal was a $986-\mathrm{Hz}$ pure tone, gated on and off with $2.5-\mathrm{ms}$ raised-cosine ramps ${ }^{6}$. The signal duration was either 12.5 or $87.5 \mathrm{~ms}$, measured at the half-rise points. The noise masker was either broadband $(250$ to $4000 \mathrm{~Hz})$ or narrowband $(923$ to $1051 \mathrm{~Hz})$. Prior to each threshold estimation run, a 10.7-s masker sample was generated in the frequency domain by setting the magnitude of components outside the desired passband to zero. This sample was played continuously throughout the course of a threshold estimation track. Prior to modulation, the narrowband masker level was $50 \mathrm{~dB}$ SPL, and the broadband masker level was $65 \mathrm{~dB}$ SPL. Modulation reduced these levels by just over $3 \mathrm{~dB}$.

The masker envelope was manipulated using 2.5-ms raised-cosine gates that were controlled using software running on the real-time digital signal processor (RP2; Tucker-Davis Technologies). For the periodic-AM pattern, the masker was gated on and off at a rate of 40Hz. Masker modulation in the jittered-AM condition was identical to that in the periodicAM condition, except that the duration associated with each phase of a modulation period (maximum and subsequent minimum) was randomly selected from a uniform distribution spanning 2.5 to $22.5 \mathrm{~ms}$. Modulation jitter was suspended for either one or four periods in each listening interval, coinciding with time points when the 12.5 - or $87.5-\mathrm{ms}$ signal might be presented. When jitter was suspended, the AM period was fixed at $12.5 \mathrm{~ms}$ per phase, consistent with the modulation pattern of the periodic-AM condition. The onset of the signal coincided with the offset of a masker envelope maximum, such that the 12.5 -ms signal was temporally centered in a single modulation minimum, and the $87.5-\mathrm{ms}$ signal spanned four modulation minima. The relationship between the signal timing and masker modulation is illustrated in Figure 4.

Each listening interval was $200 \mathrm{~ms}$ in duration, and the inter-stimulus interval was $500 \mathrm{~ms}$ in duration. Signal level was adjusted using a 3-down, 1-up procedure, estimating the signal level associated with 79\% correct (Levitt, 1971). Initially the signal level was adjusted in steps of $4 \mathrm{~dB}$; steps were reduced to $2 \mathrm{~dB}$ after the second track reversal. A track continued until 8 reversals had been obtained, and threshold estimates were computed as the mean signal level at the last 6 reversals. At least three such estimates were obtained, blocked by condition, with a fourth in cases where the first three spanned a range of $3 \mathrm{~dB}$ or more. Conditions were completed in quasi-random order. All simple effects tests used a Bonferroni correction.

\subsection{Results}

The pattern of results was consistent across individual listeners, so the mean data are shown in Figure 5. Thresholds for signals in the narrowband masker are represented with filled triangles and dotted lines; thresholds for signals in the broadband masker are represented with open circles and solid lines. Results obtained using the steady masker, shown with horizontal lines, are described first.

\footnotetext{
${ }^{6}$ The use of 2.5-ms ramps for gating the signal and masker ensured that the masker was $100 \%$ modulated, even for the briefest AM minimum of $2.5 \mathrm{~ms}$.
} 
3.3.1. Thresholds for Signals in the Steady Masker-Thresholds for signals in the steady broadband masker improved on average by $8.8 \mathrm{~dB}$ with increasing signal duration, representing a rate of $3.1 \mathrm{~dB}$ per doubling in duration. In contrast, temporal integration for signals in the narrowband masker was only $1.3 \mathrm{~dB}$, representing $0.5 \mathrm{~dB}$ per doubling in duration. As discussed in the context of the previous experiment, less than the expected temporal integration in the steady narrowband masker could be related to cues based on spectral splatter.

3.3.2. Effects of Masker AM (MUD)—The introduction of masker AM reduced thresholds for signals in the broadband masker for both jittered and periodic AM; recall that some of this benefit could be related to the 3 -dB reduction in masker level association with masker modulation. For signals in the narrowband masker, the introduction of masker AM improved thresholds for the 87.5-ms signal conditions, but it elevated thresholds for the 12.5-ms signal. Masking release for the $87.5-\mathrm{ms}$ signal is considered first. For the $87.5-\mathrm{ms}$ signal in the broadband maskers, the MUD was similar for the jittered-AM $(12.5 \mathrm{~dB})$ and the periodic-AM $(12.7 \mathrm{~dB})$ masker.

For the narrowband masker, there was a somewhat smaller MUD for the jittered-AM ( 8.7 $\mathrm{dB})$ than for the periodic-AM $(10.9 \mathrm{~dB})$ masker conditions. A repeated-measures ANOVA was performed to assess this pattern of results, with the factors masker bandwidth (broad, narrow) and envelope regularity (jittered-AM, periodic-AM). There was a main effect of bandwidth $\left(\mathrm{F}_{1,8}=49.12, \mathrm{p}<0.001\right)$, a main effect of masker envelope regularity $\left(\mathrm{F}_{1,8}=18.52\right.$, $\mathrm{p}=0.003)$, and an interaction between bandwidth and envelope regularity $\left(\mathrm{F}_{1,8}=11.15\right.$, $\mathrm{p}=0.010$ ). Simple effects tests confirmed no effect of masker AM jitter for the broadband masker $(\mathrm{p}=0.465)$ and a smaller MUD for the jittered-AM than for the periodic-AM narrowband masker $(-2.1 \mathrm{~dB} ; \mathrm{p}=0.003)$.

The effect of envelope jitter on the MUD observed with the 12.5-ms signal was broadly similar to that observed with the 87.5 -ms signal. For the broadband masker, the MUD for the $12.5-\mathrm{ms}$ signal was similar for the jittered-AM $(11.3 \mathrm{~dB})$ and the periodic-AM $(11.3 \mathrm{~dB})$ masker conditions. For the narrowband condition, there was a negative MUD for both the jittered-AM $(-6.4 \mathrm{~dB})$ and the periodic-AM $(-3.6 \mathrm{~dB})$ masker conditions, despite the fact that masker AM reduced the overall masker level. A repeated-measures ANOVA indicated a main effect of bandwidth $\left(\mathrm{F}_{1,8}=1250.18, \mathrm{p}<0.001\right)$, a main effect of envelope regularity $\left(\mathrm{F}_{1,8}=9.13, \mathrm{p}=0.016\right)$, and a significant interaction $\left(\mathrm{F}_{1,8}=8.05, \mathrm{p}=0.022\right)$. Simple effects tests revealed that the effect of masker AM jitter was not significant for the broadband masker $(\mathrm{p}=0.93)$, and there was a smaller MUD for the jittered-AM than for the periodic-AM narrowband masker $(-2.8 \mathrm{~dB} ; \mathrm{p}=0.003)$. Recall the proposal that spectral splatter could have provided a cue for detecting the 12.5 -ms signal in the steady narrowband masker but not in the AM maskers, thereby reducing the estimates of MUD; the availability of a splatter cue could play a role in the negative MUD observed for the narrowband AM maskers, but it would have had the same effect in the jittered-AM and the periodic-AM conditions.

For both signal durations, the regularity of masker AM had no effect on the MUD obtained in the broadband masker conditions, but the MUD was somewhat greater (less negative) in the periodic-AM than the jittered-AM conditions for the narrowband noise masker. This effect of envelope regularity for the narrowband masker was somewhat greater for the 12.5ms signal than for the 87.5 -ms signal (effects of 2.8 vs. $2.1 \mathrm{~dB}$, respectively). It is of interest to determine whether the effect of AM regularity of the narrowband noise masker was significantly different for the two signal durations. To that end, a repeated-measures ANOVA was performed with the factors masker envelope regularity (jittered-AM, periodicAM) and signal duration $(12.5,87.5 \mathrm{~ms})$. There was a main effect of envelope regularity $\left(\mathrm{F}_{1,8}=40.56, \mathrm{p}<0.001\right)$ and a main effect of signal duration $\left(\mathrm{F}_{1,8}=1172.35, \mathrm{p}<0.001\right)$, but no 
interaction between envelope regularity and signal duration $\left(\mathrm{F}_{1,8}=0.51, \mathrm{p}=0.49\right)$. The failure to find a significant interaction between signal duration and envelope regularity is consistent with the hypothesis that the effect of masker AM regularity is not more pronounced for the shorter signal duration. This result provides support for the assumption that envelope effects observed with a brief signal also apply to longer signals.

3.3.3. Effects of AM Masker Bandwidth (CMR)-For the 87.5-ms signal, the CMR was modest, with means of $2.3 \mathrm{~dB}$ for the jittered-AM and $0.3 \mathrm{~dB}$ for the periodic-AM maskers. The CMR was substantially larger for the 12.5 -ms signal conditions, with values of $8.7 \mathrm{~dB}$ for the jittered-AM and $6.0 \mathrm{~dB}$ for the periodic-AM maskers. A repeated-measures ANOVA indicated there was a main effect of envelope regularity $\left(\mathrm{F}_{1,8}=20.23, \mathrm{p}=0.002\right)$, a main effect of signal duration $\left(\mathrm{F}_{1,8}=112.14, \mathrm{p}<0.001\right)$, but no interaction between envelope regularity and signal duration $\left(\mathrm{F}_{1,8}=0.39, \mathrm{p}=0.547\right)$. For both durations, the $\mathrm{CMR}$ was greater in the jittered-AM than the periodic-AM masker conditions, due to relatively poor performance in the narrowband jittered-AM condition.

3.3.4. Effects of Duration for Signals in AM Maskers-Temporal integration for a signal in the broadband masker was $10.2 \mathrm{~dB}$ in the periodic-AM and $10.1 \mathrm{~dB}$ in the jitteredAM masker condition. These values represent approximately $3.6 \mathrm{~dB}$ per doubling of signal duration. If integration were acting on cues associated with listening in the dips, then a 10$\mathrm{dB}$ decrease in threshold would represent $5 \mathrm{~dB}$ per doubling in duration (1 vs 4 minima).

Temporal integration for a signal in a narrowband masker was $15.9 \mathrm{~dB}$ in the periodic-AM and $16.5 \mathrm{~dB}$ in the jittered-AM masker conditions. This represents approximately $5.7 \mathrm{~dB}$ per doubling of signal duration. The finding of relatively large integration for the narrowband AM could be interpreted as evidence that spectral splatter played little or no role in the results for these conditions. The splatter cues at signal onset and offset would be comparable for the two signal durations, so temporal integration should be reduced or eliminated by reliance on these cues.

\subsection{Discussion}

One goal of the present experiment was to determine whether effects of modulation adaptation could be observed when the masker was played continuously over the course of the threshold estimation track, and the jittered envelope lacked a prominent spectral component at the standard envelope rate. Recall that modulation jitter was suspended during presentation of the signal, so energetic masking was equivalent in the periodic and jittered envelope conditions. Thresholds for signals in the broadband masker were not significantly different in the jittered-AM and periodic-AM conditions for either the 12.5- or $87.5-\mathrm{ms}$ signal. This provides further support for the idea that the 200-ms signal data in Experiment 1 and those of Verhey and Ernst (2009) reflect the introduction of relatively long-duration AM minima rather than modulation adaptation.

Another goal of this experiment was to assess what role signal duration might play in the effect of masker envelope regularity for the broadband masker. The effect of envelope regularity on the MUD did not differ for the 12.5- and $87.5-\mathrm{ms}$ signal conditions. Whereas there was no effect of envelope regularity for the broadband masker, thresholds were higher and the MUD was smaller in the jittered-AM than the periodic-AM narrowband noise conditions. There is precedent for this finding in the results of Buss et al. (2009). In that study, detection thresholds for a brief signal in a narrowband masker were lower when the masker envelope was periodic than when its rate was changed unpredictably before and after the signal interval. One interpretation of this result is that listeners benefit from a regular 
envelope pattern when the masker is spectrally narrow. Deviations from this regular pattern could serve as a cue to the presence of the signal.

The CMRs obtained with both the jittered-AM and periodic-AM maskers were about $6 \mathrm{~dB}$ larger for the $12.5-\mathrm{ms}$ than for the $87.5-\mathrm{ms}$ signal. Some studies of CMR have found greater masking release for long than short signals (Buus et al., 1996), although this is not always the case (Hicks and Bacon, 1995). For example, Hicks and Bacon (1995) measured tone detection in a masker that was composed of one or more sinusoidally amplitude modulated tones. The CMR in that study was larger when the signal was a single burst, coincident with an AM minimum, than when it was a series of three bursts, coincident with three consecutive AM minima. As in the present data, Hicks and Bacon (1995) found substantially more than $3 \mathrm{~dB}$ of temporal integration per doubling of signal duration when thresholds were estimated with a single narrowband masker. They proposed that detection of the threeburst signal was based on a change in envelope statistics, but that this cue was unavailable to the listener with the single-burst signal. A similar argument was proposed by Schooneveldt and Moore (1989) to account for the finding that AM applied to a narrowband masker improved performance when the signal was relatively long ( $>100 \mathrm{~ms})$ but not when it was relatively short $(<100 \mathrm{~ms})$. The present dataset is broadly consistent with this hypothesis, with the caveat that listeners in the present study were able to benefit from narrowband masker envelope periodicity even for a brief (12.5-ms) signal. One factor that may be important in differentiating studies finding greater CMR with short vs. long signals could be the consistency of masker envelope minima. Greater CMRs for longer signals would be expected for stimuli with inconsistent modulation minimum depth or duration, due to the increased probability of the signal coinciding with a 'good' minimum. This is consistent with the fact Buus et al. (1996) manipulated the coherence of noise-based envelopes and found greater CMR for long signals, whereas Hicks and Bacon (1995) used sinusoidally amplitude modulated tones as maskers and found greater CMR for short signals.

An unexpected finding of the present experiment is the relatively large temporal integration observed for the broadband, AM maskers. Based on a multiple looks model (Viemeister and Wakefield, 1991), increasing the signal duration from 12.5- to 87.5-ms was expected to improve thresholds by $6 \mathrm{~dB}$, due to the factor-of-four increase in the number of masker modulation minima containing the signal. Integration was approximately $10 \mathrm{~dB}$ for both envelope patterns, however. While greater than expected integration has been observed previously for signals in comodulated maskers, where AM was due to inherent fluctuation of a narrowband noise or to multiplication with a lowpass noise (Buus, 1999; Schooneveldt and Moore, 1989), it was not expected in the present paradigm. Noise-based AM is associated with variability in the depth and duration of modulation minima, so greater integration in those maskers could reflect the increased probability of the signal coinciding with a 'good' masker minimum. The use of square-wave modulation in the current study was predicted to provide more uniform opportunities to detect the signal. Stimuli used in the present experiment share some salient features with the stimuli used by Viemeister and Wakefield (1991). In that study, temporal integration was measured for a pair of 10-ms tone bursts presented in 10-ms gaps of noise, separated by $100 \mathrm{~ms}$. Thresholds improved by $2.5 \mathrm{~dB}$ when the number of tone bursts was increased from one to two. It is unclear what accounts for the greater-than-expected integration in AM maskers of the present study. One possibility is that signal energy outside of the modulation minima also contributed to performance. Further study is needed to more fully understand temporal integration in a coherently amplitude modulated masker. 


\section{General Discussion \& Conclusions}

The experiments reported here followed up on the finding of greater ability to benefit from broadband masker modulation with an irregular pattern than with a periodic pattern of AM, reported by Verhey and Ernst (2009). The first experiment replicated this finding with a 200 -ms tone, as used in that study, but found no benefit of envelope jitter when a 12.5 -ms signal was presented in a modulation minimum of fixed duration. This finding provides evidence against a role of adaptation for the long signal and instead supports the idea that occasional, relatively long-duration AM minima are especially beneficial for signal detection. Experiment 2 used a continuous masker and a different procedure for introducing envelope jitter, associated with a broad distribution of envelope modulation frequencies. As in the previous experiment, introducing temporal jitter to the broadband masker envelope had no reliable effect on brief-signal thresholds when the duration of masker AM minima coincident with the signal was fixed. This was true for both 12.5 - and $87.5-\mathrm{ms}$ signal durations. As in Experiment 1, there was no indication that adaptation to modulation played a role in the results.

While envelope jitter before and after the signal presentation did not affect thresholds for signals in the broadband maskers, it did affect thresholds for the signal in the narrowband maskers. Thresholds for a brief signal presented in narrowband maskers tended to be lower for the periodic-AM than the jittered-AM conditions. While this effect failed to reach significance in Experiment 1, it was significant for both signal durations in Experiment 2. One way to think about these results is in terms of informational masking. In general, informational masking is largest under conditions of stimulus uncertainty and signal/masker similarity (Durlach et al., 2003; Kidd et al., 1994; Neff, 1995). If the addition of a signal to a narrowband masker results in stimulus features that resemble the features of the masker alone, then confusion could have a significant impact on performance. The introduction of comodulated masker components remote from the signal frequency, however, could reduce this confusion, perhaps due to cued listening (Buus, 1985) or a process related to auditory grouping (Dau et al., 2005; Grose and Hall, 1993). The benefit of narrowband masker envelope regularity is likely related to the observation that non-simultaneous masking for a narrowband masker can be reduced with the introduction of off-frequency cues to masker onset and offset (Moore and Glasberg, 1982; Neff, 1986). By this view, the detection threshold for a signal in a masker that spans multiple auditory filters is a better indicator of energetic masking than threshold in a narrowband masker.

The results of both experiments reported here support the conclusion that the greater MUD with irregular than a periodic broadband masker AM in long-duration signal conditions (Exp 1 of the present report; Verhey and Ernst, 2009) is due to the introduction of relatively long duration AM minima. When jitter was suspended during the signal presentation, no effect of envelope regularity was observed with broadband maskers. In contrast, envelope regularity appeared to benefit detection in the narrowband masker under some conditions, a result that was interpreted in terms of stimulus uncertainty and informational masking. One challenge for future modeling efforts will be to capture these narrowband masker effects. Additional work is also needed to understand the effects of signal duration on the ability to benefit from broadband masker AM.

\section{Acknowledgments}

This work was supported by NIH NIDCD RO1-DC007391. Detailed feedback on this report was provided by Brian Moore and two anonymous reviewers. 


\section{Abbreviations}
AM
amplitude modulation
CMR
comodulation masking release
MUD
modulated-unmodulated difference

\section{References}

ANSI. ANSI S3.6-2010, American National Standard Specification for Audiometers. American National Standards Institute; New York: 2010.

Bacon SP, Lee J, Peterson DN, Rainey D. Masking by modulated and unmodulated noise: Effects of bandwidth, modulation rate, signal frequency, and masker level. J Acoust Soc Am. 1997; 101:16001610. [PubMed: 9069628]

Bruckert L, Herrmann M, Lorenzi C. No adaptation in the amplitude modulation domain in trained listeners. J Acoust Soc Am. 2006; 119:3542-3545. [PubMed: 16838495]

Buss E, Grose JH, Hall JW. Features of across-frequency envelope coherence critical for comodulation masking release. J Acoust Soc Am. 2009; 126:2455-2466. [PubMed: 19894826]

Buus S. Release from masking caused by envelope fluctuations. J Acoust Soc Am. 1985; 78:19581965. [PubMed: 4078172]

Buus S. Temporal integration and multiple looks, revisited: Weights as a function of time. J Acoust Soc Am. 1999; 105:2466-2475. [PubMed: 10212427]

Buus S, Zhang L, Florentine M. Stimulus-driven, time-varying weights for comodulation masking release. J Acoust Soc Am. 1996; 99:2288-2297. [PubMed: 8730075]

Carlyon RP, Buus S, Florentine M. Comodulation masking release for three types of modulator as a function of modulation rate. Hear Res. 1989; 42:37-45. [PubMed: 2584158]

Dau T, Kollmeier B, Kohlrausch A. Modeling auditory processing of amplitude modulation. I Detection and masking with narrow-band carriers. J Acoust Soc Am. 1997; 102:2892-2905. [PubMed: 9373976]

Dau, T.; Ewert, SD.; Oxenham, AJ. Effects of concurrent and sequential streaming in comodulation masking release. In: Pressnitzer, D.; de Cheveigne, A.; McAdams, S.; Collet, L., editors. Auditory Signal Processing: Physiology, Psychoacoustics and Models. Springer Verlag; 2005. p. 335-343.

Durlach NI, Mason CR, Shinn-Cunningham BG, Arbogast TL, Colburn HS, Kidd G Jr. Informational masking: Counteracting the effects of stimulus uncertainty by decreasing target-masker similarity. J Acoust Soc Am. 2003; 114:368-379. [PubMed: 12880048]

Garner WR, Miller GA. The masked threshold of pure tones as a function of duration. J Exp Psychol. 1947; 37:293-303. [PubMed: 20258425]

Gerken GM, Bhat VK, Hutchison-Clutter M. Auditory temporal integration and the power function model. J Acoust Soc Am. 1990; 88:767-778. [PubMed: 2212302]

Grose JH, Hall JW. Comodulation masking release using SAM tonal complex maskers: Effects of modulation depth and signal position. J Acoust Soc Am. 1989; 85:1276-1284. [PubMed: 2708670]

Grose JH, Hall JW. Comodulation masking release: Is comodulation sufficient? J Acoust Soc Am. 1993; 93:2896-2902. [PubMed: 8315153]

Hicks ML, Bacon SP. Some factors influencing comodulation masking release and across-channel masking. J Acoust Soc Am. 1995; 98:2504-2514. [PubMed: 7593933]

Kawashima T. Attention modulates auditory adaptation produced by amplitude modulation. J Acoust Soc Am. 2009; 126:EL123-127. [PubMed: 19894786]

Kay RH, Matthews DR. On the existence in human auditory pathways of channels selectively tuned to the modulation present in frequency-modulated tones. J Physiol. 1972; 225:657-677. [PubMed: 5076392]

Kidd G Jr, Mason CR, Deliwala PS, Woods WS, Colburn HS. Reducing informational masking by sound segregation. J Acoust Soc Am. 1994; 95:3475-3480. [PubMed: 8046139] 
Levitt H. Transformed up-down methods in psychoacoustics. J Acoust Soc Am. 1971; 49:467-477. [PubMed: 5541744]

McFadden D. Comodulation masking release: Effects of varying the level, duration, and time delay of the cue band. J Acoust Soc Am. 1986; 80:1658-1667. [PubMed: 3794071]

Moore BCJ, Glasberg BR. Contralateral and ipsilateral cueing in forward masking. J Acoust Soc Am. 1982; 71:942-945. [PubMed: 7085981]

Moore BCJ, Fullgrabe C, Sek A. Estimation of the center frequency of the highest modulation filter. J Acoust Soc Am. 2009; 125:1075-1081. [PubMed: 19206882]

Moore BCJ, Glasberg BR, Plack CJ, Biswas AK. The shape of the ear's temporal window. J Acoust Soc Am. 1988; 83:1102-1116. [PubMed: 3356815]

Neff DL. Confusion effects with sinusoidal and narrow-band noise forward maskers. J Acoust Soc Am. 1986; 79:1519-1529. [PubMed: 3711451]

Neff DL. Signal properties that reduce masking by simultaneous, random-frequency maskers. J Acoust Soc Am. 1995; 98:1909-1920. [PubMed: 7593915]

Richards VM, Buss E, Tian L. Effects of modulator phase for comodulation masking release and modulation detection interference. J Acoust Soc Am. 1997; 102:468-476. [PubMed: 9228809]

Schooneveldt GP, Moore BCJ. Comodulation masking release (CMR) as a function of masker bandwidth, modulator bandwidth, and signal duration. J Acoust Soc Am. 1989; 85:273-281. [PubMed: 2921409]

Tansley BW, Suffield JB. Time course of adaptation and recovery of channels selectively sensitive to frequency and amplitude modulation. J Acoust Soc Am. 1983; 74:765-775. [PubMed: 6630734]

van den Brink WA, Houtgast T. Spectro-temporal integration in signal detection. J Acoust Soc Am. 1990; 88:1703-1711. [PubMed: 2262627]

Verhey JL, Ernst SM. Comodulation masking release for regular and irregular modulators. Hear Res. 2009; 253:97-106. [PubMed: 19341786]

Verhey JL, Dau T, Kollmeier B. Within-channel cues in comodulation masking release (CMR): Experiments and model predictions using a modulation-filterbank model. J Acoust Soc Am. 1999; 106:2733-2745. [PubMed: 10573889]

Viemeister NF, Wakefield GH. Temporal integration and multiple looks. J Acoust Soc Am. 1991; 90:858-865. [PubMed: 1939890]

Wojtczak M, Viemeister NF. Suprathreshold effects of adaptation produced by amplitude modulation. J Acoust Soc Am. 2003; 114:991-997. [PubMed: 12942978]

Wojtczak M, Viemeister NF. Forward masking of amplitude modulation: basic characteristics. J Acoust Soc Am. 2005; 118:3198-3210. [PubMed: 16334900] 


\section{Highlights}

- Introducing jitter to the masker envelope pattern can affect the detection threshold for a pure-tone signal.

- Effects of masker irregularity are not found when jitter is suspended during presentation of the signal.

- Results are more consistent with local envelope rate effects than modulation adaptation. 


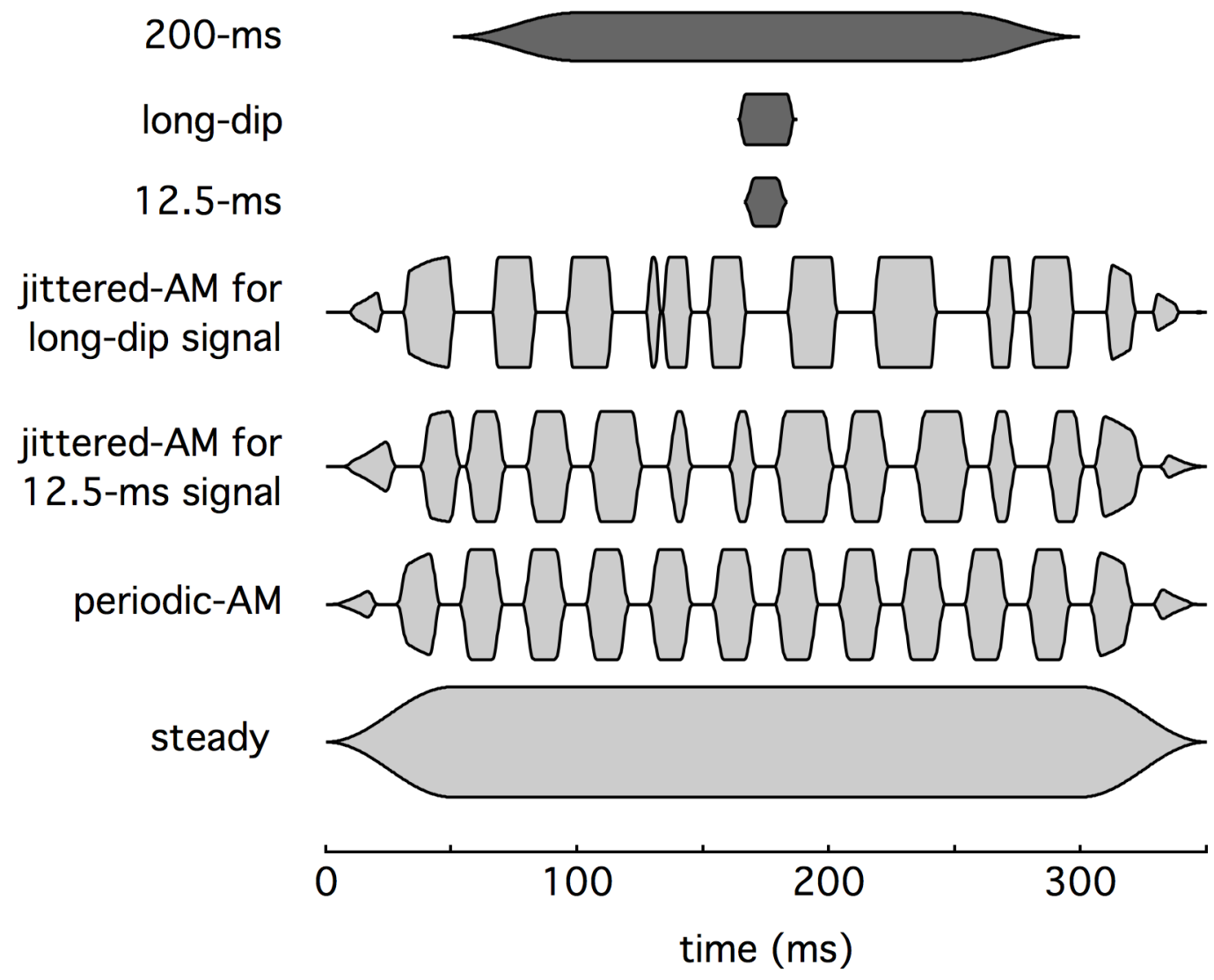

Fig. 1.

Basic features of the temporal envelopes used in Experiment 1 are illustrated as a function of time. Signals are illustrated with dark fill, and masker envelope patterns are illustrated with light fill. Notice that the 12.5 -ms signal is coincident with and equal in duration to a modulation minimum in both the periodic- $A M$ and jittered-AM maskers. The long-dip signal is coincident with and equal in duration to a modulation minimum in the associated jittered- $A M$ masker. 


\section{Signal condition}

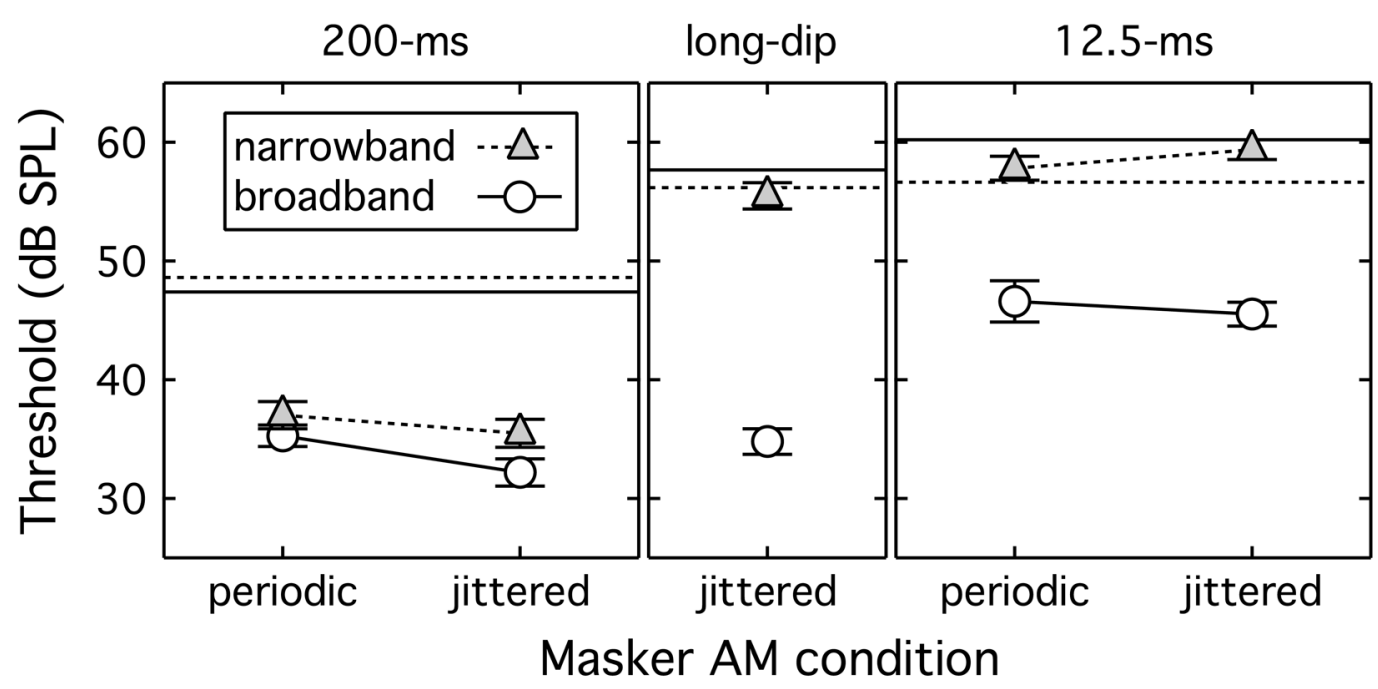

Fig. 2.

Mean thresholds are plotted in dB SPL for each masker condition, with results from the three signal conditions in separate panels. Open circles and solid lines indicate mean thresholds in the broadband masker conditions. Filled triangles and dotted lines indicate mean thresholds in the narrowband masker conditions. Error bars show \pm 1 standard error of the mean. Standard errors for the steady masker conditions (not shown) were between 0.9 and $1.7 \mathrm{~dB}$. 


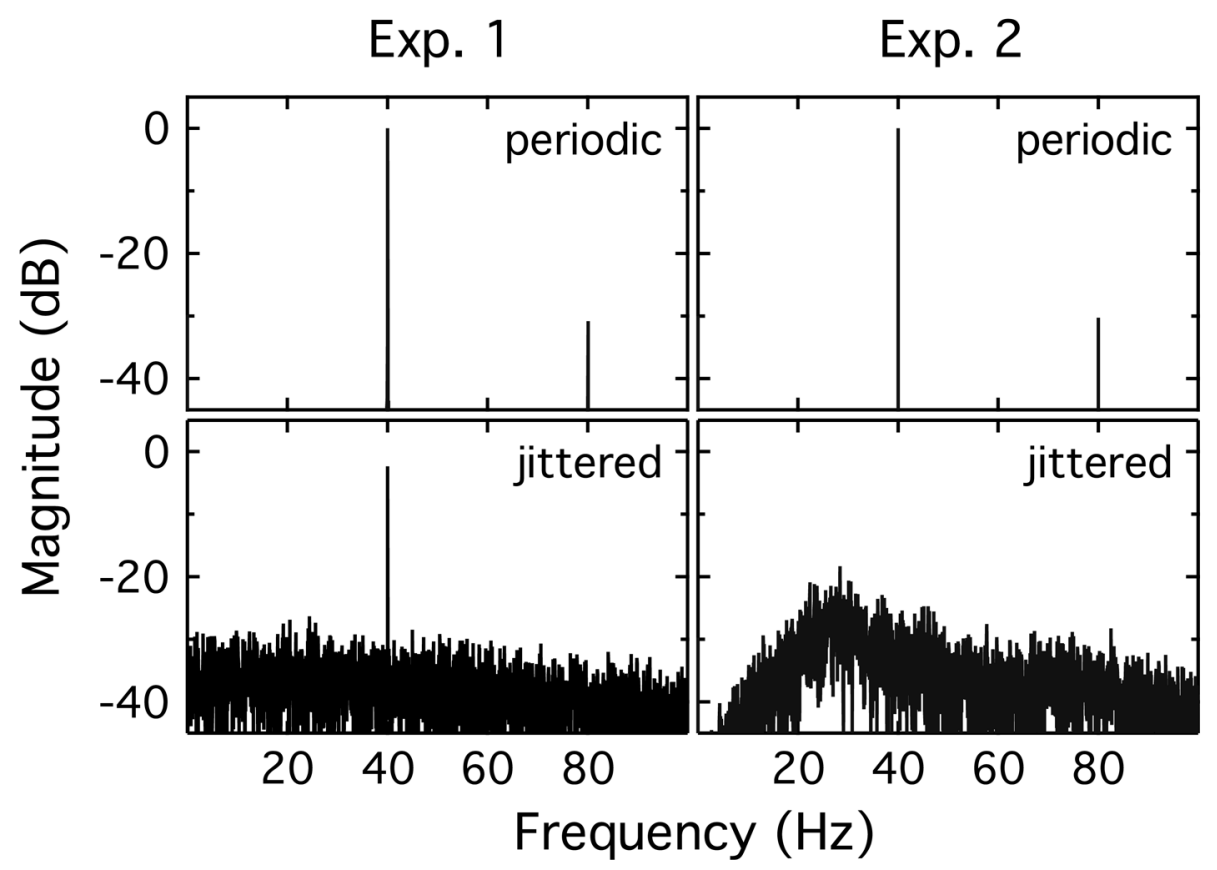

Fig. 3.

Magnitude spectra for the periodic- $A M$ and jittered- $A M$ conditions in each experiment are shown in the top and bottom panels, respectively. Stimuli for Experiment 1 are shown in the left column, and those for Experiment 2 are shown in the right column. Magnitude is plotted in $\mathrm{dB}$ relative to the magnitude at the peak modulation frequency in the periodic- $A M$ condition. Frequency was computed with a resolution of $0.1 \mathrm{~Hz}$. 


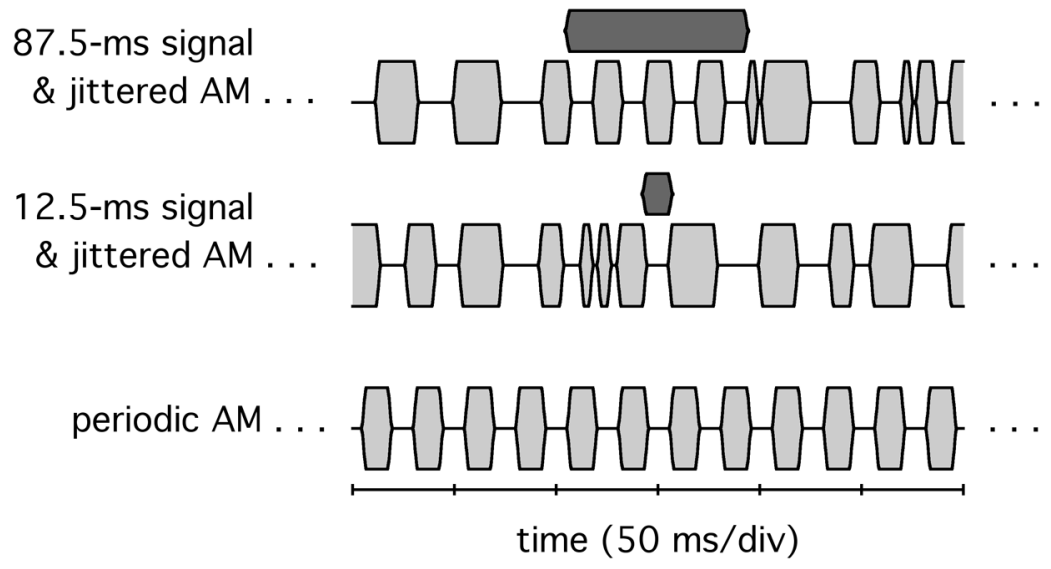

Fig. 4.

Basic features of the temporal envelopes used in Experiment 2 are illustrated as a function of time, following the conventions of Fig 1 . Two examples of the jittered-AM envelope are shown: one appropriate for the 87.5-ms signal and one appropriate for the 12.5-ms signal. Notice that modulation jitter is suspended for AM periods associated with the signals. 


\section{Signal condition}

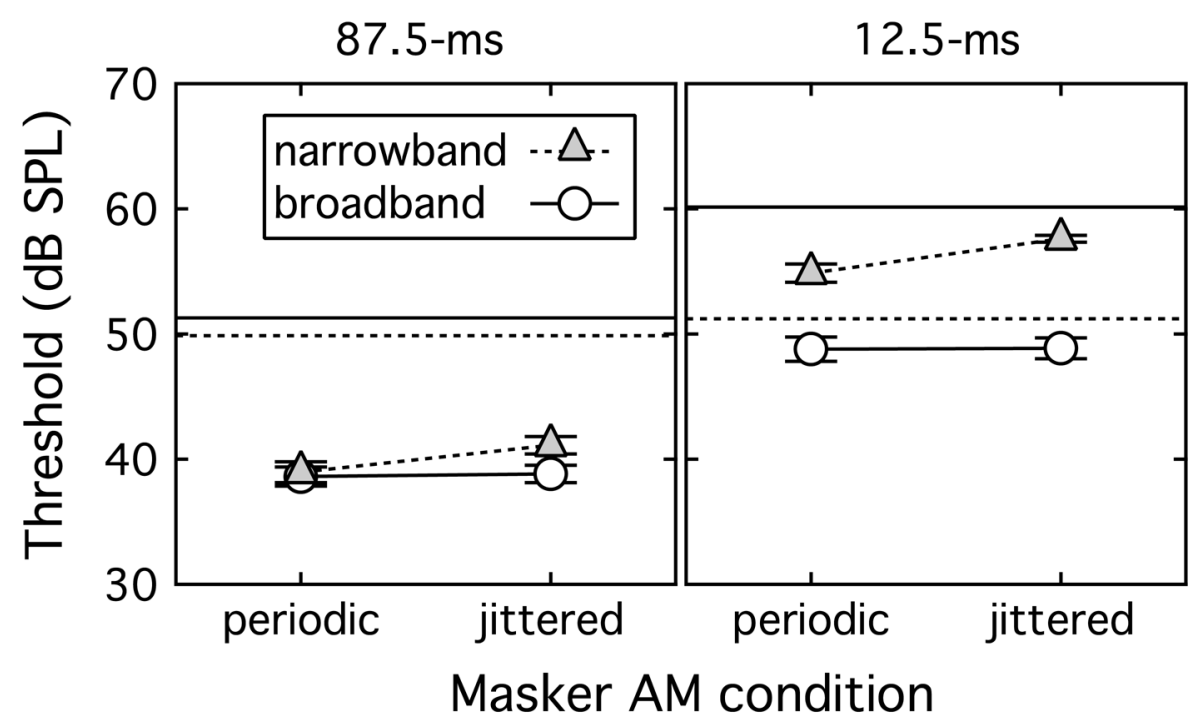

Fig. 5.

Mean thresholds are plotted in dB SPL for each masker condition, with results for the two signal conditions in separate panels. The plotting conventions follow those of Fig 2. Error bars show \pm 1 standard error of the mean. Standard errors for the steady masker conditions (not shown) were between 0.7 and $1.0 \mathrm{~dB}$. 